\title{
COVID-19 pneumonia: The first two chest CTs in the Bamrasnaradura Infectious Disease Institute
}

\author{
Panyaros Kongpanya, M.D. ${ }^{(1)}$ \\ Parichut Vongthawatchai, M.D. ${ }^{(1)}$ \\ Priyanut Atiburanakul, M.D. ${ }^{(1)}$ \\ Nayot Panitanum, M.D. ${ }^{(2)}$ \\ Patama Suttha, M.D. ${ }^{(2)}$ \\ From ${ }^{(1)}$ Department of Radiology, Bamrasnaradura Infectious Disease Institute, \\ Nonthaburi, Thailand. \\ ${ }^{(2)}$ Department of Internal Medicine, Bamrasnaradura Infectious Disease Institute, \\ Nonthaburi, Thailand. \\ Address correspondence to P.K. (e-mail: panyaros056@gmail.com)
}

\section{Abstract}

Coronavirus disease 2019 (COVID-19), caused by the severe acute respiratory syndrome coronavirus 2 (SARS-CoV-2), continues to spread rapidly around the world. We reported the first two cases of COVID-19 pneumonia who had the chest computed tomography (CT) performed at the Bamrasnaradura Infectious Disease Institute (BIDI). The chest CT findings in the two patients with COVID-19 pneumonia showed bilateral lung involvement, multifocal involvement, peripheral distribution, ground glass opacity (GGO), consolidation and GGO with interlobular septal thickening ("crazy-paving" pattern). The chest CT findings in these patients are nonspecific and overlapped with other diseases.

Keywords: COVID-19, SARS-CoV-2, Chest computed tomography, Ground glass opacity, Consolidation. 


\section{Introduction}

In the late 2019, a large outbreak of pneumonia with an unknown cause was reported in Wuhan, Hubei province, China. A novel coronavirus, officially named severe acute respiratory syndrome coronavirus 2 (SARS-CoV-2), was later identified as the cause of the outbreak, named coronavirus disease 2019 (COVID-19) by the World Health Organization (WHO)[1], which is currently spreading in several countries around the world.

\section{Case Summary}

\section{Case 1}

A 35-year-old female is a health-care worker with Hemoglobin E trait. Four days before her admission to a private hospital in Bangkok, she had close contact with a COVID-19 patient at her workplace and developed a low-grade fever and a cough during the next 2 days. The result of the real-time reverse transcription polymerase chain reaction (RT-PCR) detected SARS-CoV-2 on the date of the initial symptoms. The initial chest radiograph (CXR), 2 days after the initial symptoms, was normal. She received a treatment with lopinavir/ritonavir, oseltamivir, and ganciclovir. Seven days after the initial symptoms, her CXR started to show the first abnormalities and she received treatments with chloroquine, darunavir, and ritonavir instead. Nine days after the initial symptoms, her clinical symptoms progressed and the CXR showed an increase in abnormalities. Therefore, she was referred to the Bamrasnaradura Infectious Disease Institute (BIDI) for further treatment. Her physical examination revealed the body temperature of $37.9^{\circ} \mathrm{C}$, the pulse of 110 beats per minute (bpm), a normal respiratory rate and blood pressure. The oxygen saturation was $95 \%$ under ambient air. Her complete blood count $(\mathrm{CBC})$ revealed normal. The CXR made at the BIDI, 9 days after the initial symptoms, showed patchy opacities at the left perihilar region, left lower lung zone, and reticular opacities at the right perihilar region, and the right lower lung zone (Figure 1). 


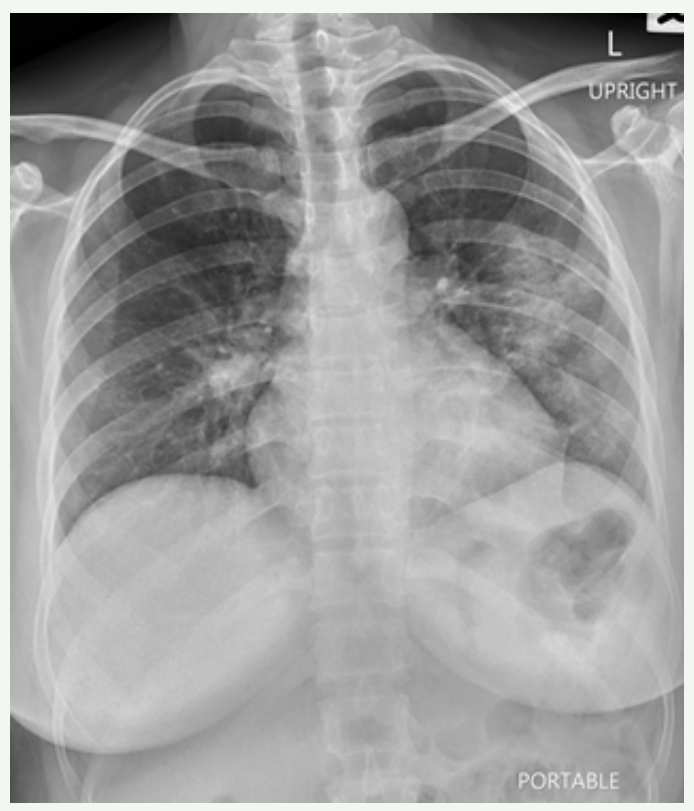

Figure 1. Frontal anteroposterior (AP) CXR shows patchy opacities at the left perihilar region and the left lower lung zone and reticular opacities at the right perihilar region and the right lower lung zone.

At the BIDI, she received treatments with favipiravir, darunavir, ritonavir, and chloroquine. Her fever resolved within 2 days. Seventeen days after the initial symptoms, she obtained a negative real-time RT-PCR result for SARS-CoV-2. However, the CXR showed an increase of opacities (Figure 2).

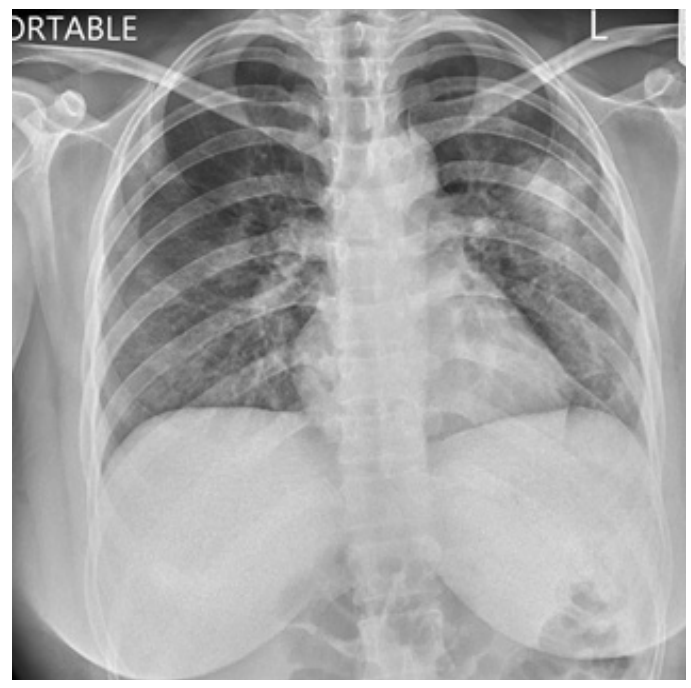

Figure 2. Frontal AP CXR shows an increase of patchy opacities with ground glass opacities (GGO) at the left perihilar region and the left lower lung zone, and an increase of reticular opacities with GGO at the right perihilar region and the right lower lung zone. 
The non-contrast chest computed tomography (CT) was performed for evaluation, 18 days after the initial symptoms, which showed GGO with interlobular septal thickening (crazy-paving pattern) at both lungs (Figure 3). Thirty-three days after the initial symptoms, she had no symptom, and she had a follow-up non-contrast chest CT which marked resolutions of lung abnormalities (Figure 4).

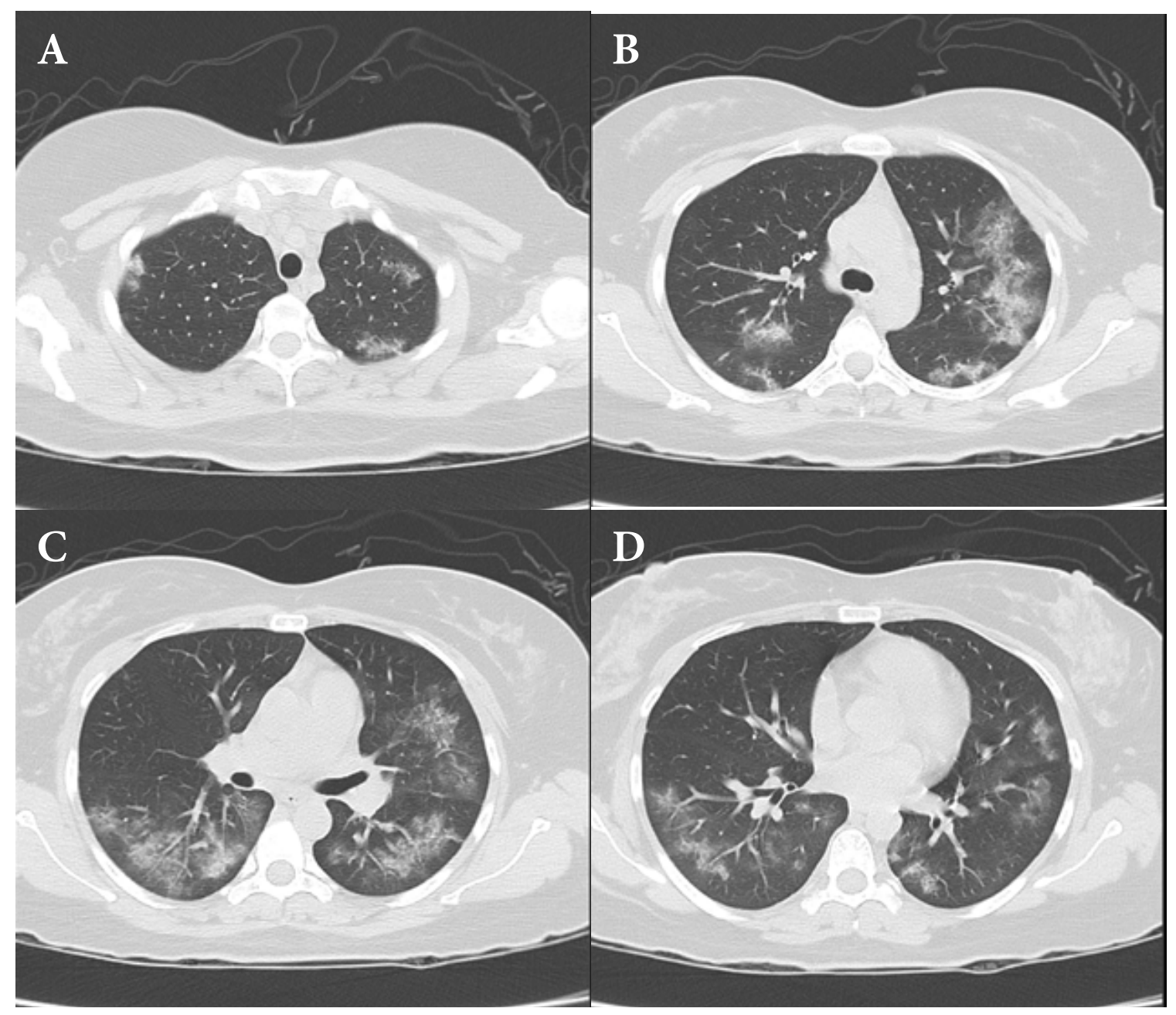

Figure 3. Axial non-contrast chest $C T(A-D)$ shows crazy-paving pattern at both upper lobes and both lower lobes, involving peripheral regions with presence of subpleural sparing. 


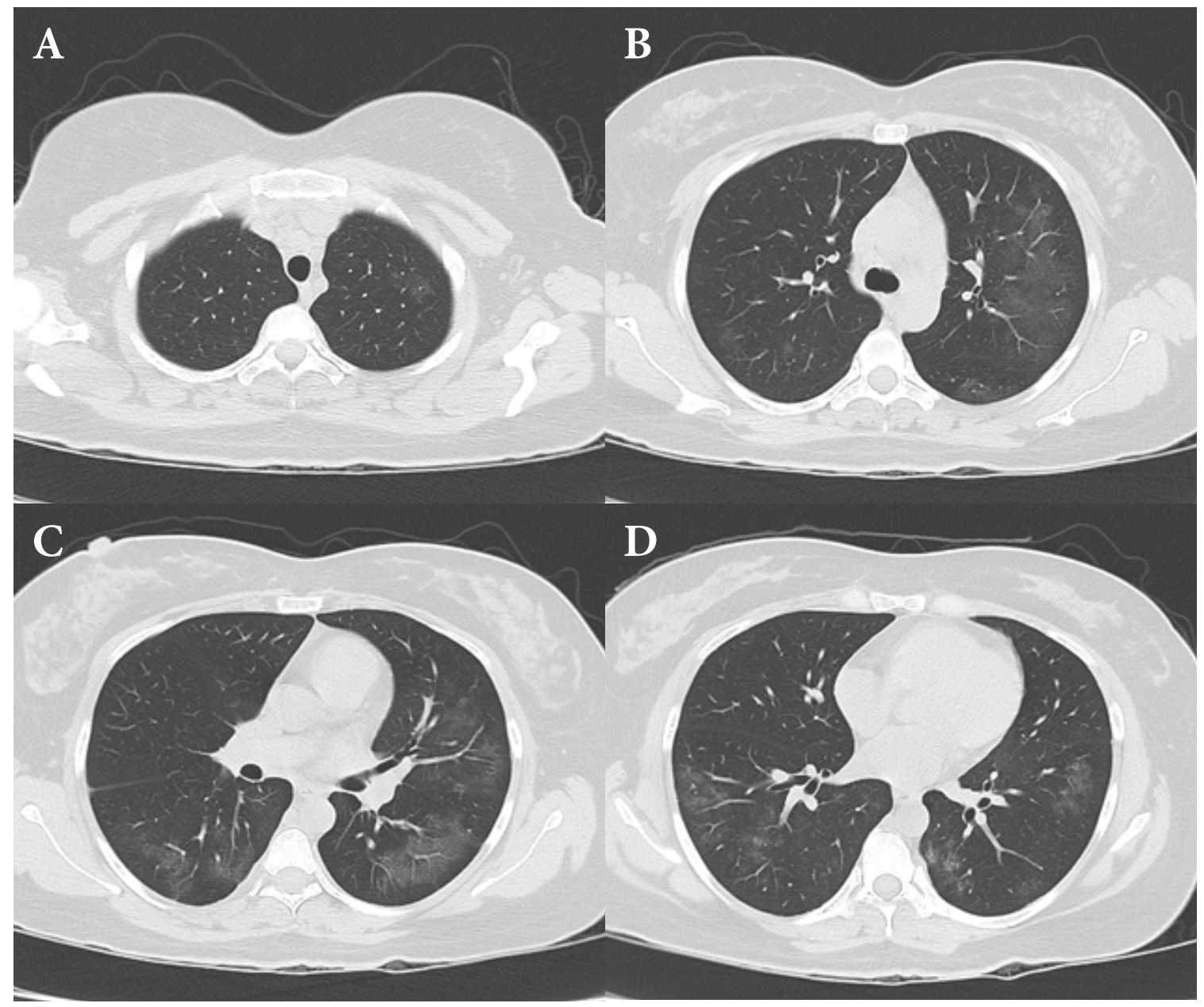

Figure 4. Axial non-contrast chest $C T(A-D)$ shows resolutions of crazy-paving pattern with residual GGO at both lungs. 


\section{Case 2}

A 25-year-old male presented with a sore throat and a cough for 4 days and a fever for 1 day before admission. He returned from the Republic of Korea 2 days before the initial symptoms. His physical examination revealed the body temperature of $38.2{ }^{\circ} \mathrm{C}$, the pulse of $112 \mathrm{bpm}$, a normal respiratory rate and blood pressure. The oxygen saturation was $100 \%$ under ambient air. His CBC was normal. The result of the real-time RT-PCR detected SARS-CoV-2 and the initial CXR was a questionable abnormality at the right upper lobe, 4 days after the initial symptoms, (Figure 5).

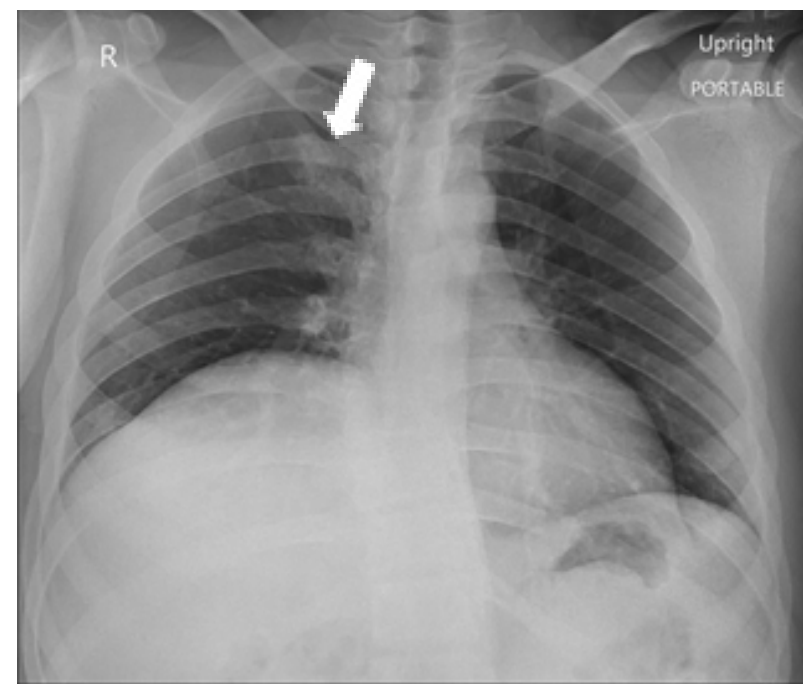

Figure 5. Frontal AP CXR shows an opacity at the right upper lobe, superimposed on the $1^{\text {st }}$ right anterior rib (arrow).

His follow-up CXR, 7 days after the initial symptoms, showed a minimal amount of GGO at the right upper lobe (Figure 6). However, he still had a fever and more dyspnea. Therefore, a non-contrast chest CT was performed in order to evaluate the severity of the disease. The non-contrast chest CT, 7 days after the initial symptoms, showed GGO at both upper lobes and both lower lobes and consolidation was visible at the right lower lobe which could not be seen on the CXR (Figure7). 


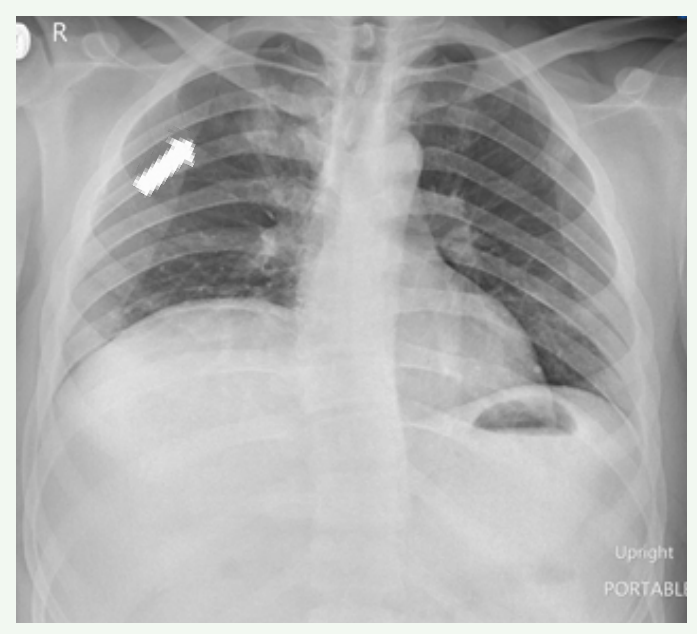

Figure 6. Frontal AP CXR shows an increase of GGO at the right upper lobe (arrow).

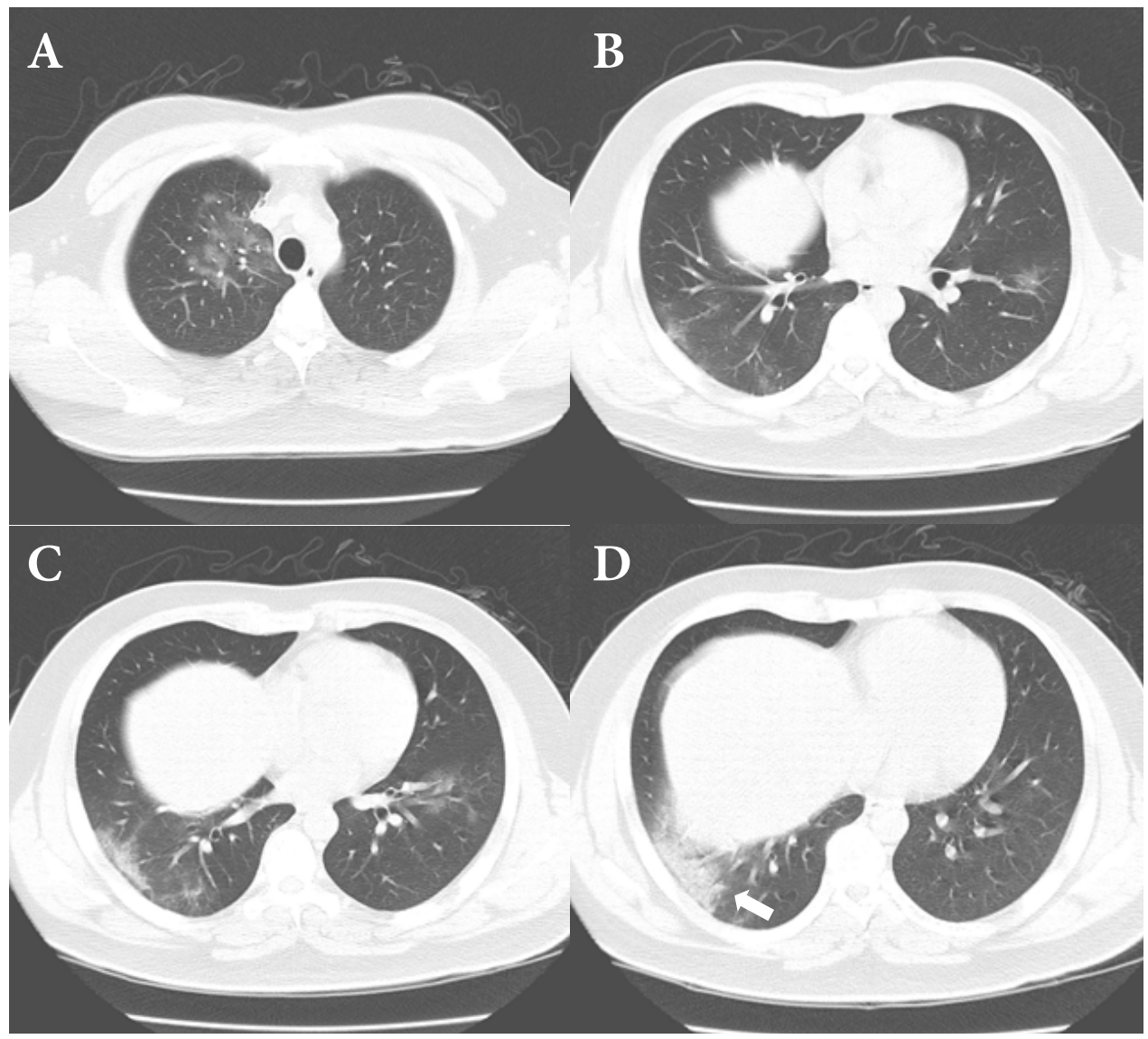

Figure 7. The axial non-contrast chest CT (A-D) shows GGO at both upper lobes and both lower lobes with subpleural consolidation (arrow) at the right lower lobe. 
After the chest CT, he received treatments with lopinavir/ritonavir, favipiravir, darunavir, ritonavir, and chloroquine and the following day, a fever was absent. The real-time RT-PCR did not detect SARS-CoV-2, 15 days after the initial symptoms. Seventeen days after the initial symptom, he had no symptom, and he was given a follow-up non-contrast chest CT, which marked resolutions of GGO at both lungs and changes of the subpleural consolidation to GGO at the right lower lobe (Figure 8).

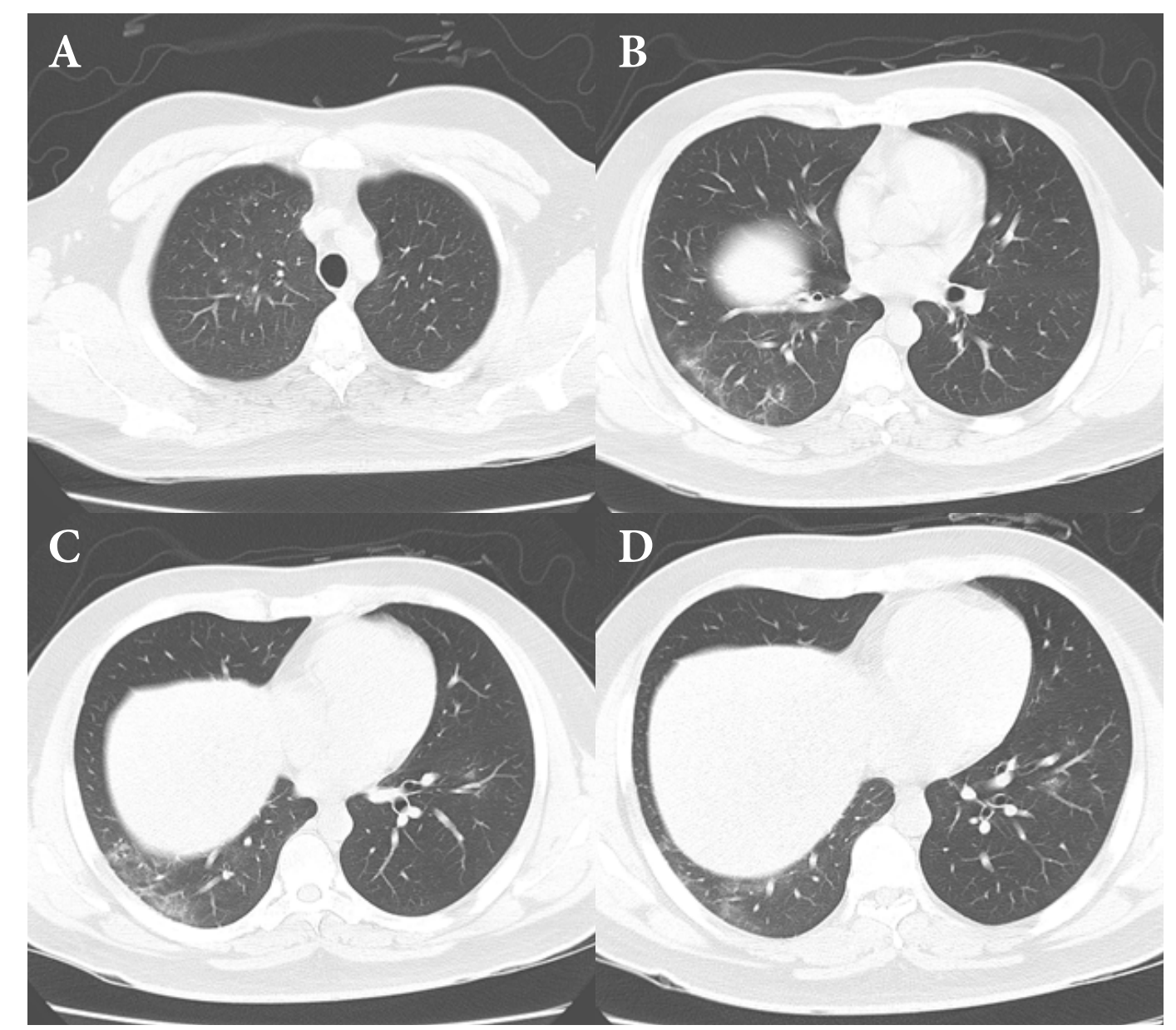

Figure 8. The axial non-contrast chest $C T(A-D)$ shows partial resolutions of $G G O$ at both upper lobes and both lower lobes. The previously seen subpleural consolidation at the right lower lobe has turned into GGO. 


\section{Discussion}

The outbreak of the COVID-19 caused by SARS-CoV-2 rapidly spreads around the world. On the $11^{\text {th }}$ of March 2020, the WHO made the assessment that the COVID-19 could be characterized as a pandemic[2]. The virus can be transmitted from person to person via droplets and close contact $[3,4]$. The patients may be asymptomatic to critical and even fatal, most patients experience mild symptoms[5]. The most common symptoms of COVID-19 are fever and cough[5-7].

The CXR is a low sensitivity modality, 69\%, in the detection of COVID-19 pneumonia in the early state[8]. The initial CXR may be normal and developed abnormalities on the follow-up CXR[8], similar to our case 1.

The chest CT findings of COVID-19 pneumonia in our cases showed bilateral and multifocal pulmonary involvements, peripheral distribution, GGO, crazy-paving pattern, absent pleural effusion and mediastinal lymphadenopathy, which are consistent with the previous studies[5, 6, 9-11]. However, the GGO and consolidation are also commonly seen in other diseases such as influenza pneumonia, adenovirus pneumonia and organizing pneumonia[12-15].

CT imaging patterns of viral pneumonia are related to the pathogenesis of viral infections. Most viral pneumonia share similar CT imaging patterns in the same families because viruses have a similar pathogenesis[12]. The outbreaks of the severe acute respiratory syndrome (SARS) in 2002-2003 and Middle East respiratory syndrome (MERS) in 2012 were caused by other strains of the coronavirus family. Therefore, chest CT findings of COVID-19 pneumonia resemble SARS and MERS, however, unilateral involvement is more common in the early acute phase of SARS and MERS[17, 18].

The follow up chest CT, 33 days and 17 days after the initial symptoms for the first case and second case respectively, showed partial resolutions of lung abnormalities. The crazy-paving pattern was no longer observed in case 1 and 
the consolidation was absorbed and turned into the GGO in case 2 which are consistent with the absorption stage in the previous study[19]. Sequelae of pulmonary fibrosis, which is likely with SARS and MERS[12, 17, 20], may in our two cases be less likely to occur, because both cases were mild cases of COVID-19 pneumonia and received early treatment with antiviral medications. There were no signs of the acute respiratory distress syndrome (ARDS) and no symptoms on the day of the follow up chest CT. Neither the crazy-paving pattern was found, probably due to the recovering stage[19]. However, detection of pulmonary fibrosis should still be followed up in the long term.

Many patients of COVID-19 were admitted at the BIDI. There were a few cases of COVID-19 pneumonia which the chest CT was performed. Although the chest CT has a high sensitivity to detect lung abnormalities and the previous studies $[5,6,10]$ suggested that the chest CT may be useful for screening or initial diagnosis of COVID-19, we do not use the chest CT screening for diagnosis of COVID-19 pneumonia, because the clinicians usually use clinical which correlates with the CXR. Therefore, it is sufficient for the diagnosis and treatment. The chest CT is mostly performed in case of a questionable abnormal CXR for an early diagnosis and treatment.

\section{Conclusion}

The chest CT findings of COVID-19 pneumonia are nonspecific and are overlapped with other viral infections and other diseases. Although the chest CT has a high sensitivity in the detection of lung abnormalities and is very useful for early diagnosis and treatment, it is not recommended as a first-choice modality to diagnose COVID-19 pneumonia. 


\section{References}

1. World Health Organization [Internet]. Geneva: WHO; c2020 [cited 2020 Mar 20]. Naming the coronavirus disease (COVID-2019) and the virus that causes it; [about 2 screens]. Available from: https://www.who.int/emergen cies/diseases/novel-coronavirus-2019/technical-guidance/naming-thecoronavirus-disease-(covid-2019)-and-the-virus-that-causes-it.

2. World Health Organization [Internet]. Geneva: WHO; 2020 [cited 2020 Mar 20]. Coronavirus disease 2019 (COVID-19) situation report - 51; [about 9 p.]. Available from: https://www.who.int/docs/default-source/coronaviruse/ situation-reports/20200311-sitrep-51-covid-19.pdf?sfvrsn=1ba62e57_10.

3. Carlos WG, Dela Cruz CS, Cao B, Pasnick S, Jamil S. Novel Wuhan (2019nCoV) coronavirus. Am J Respir Crit Care Med. 2020 Feb 15;201(4):P7-8. doi: $10.1164 / \mathrm{rccm} .2014 \mathrm{P} 7$.

4. Li Q, Guan X, Wu P, Wang X, Zhou L, Tong Y, et al. Early transmission dynamics in Wuhan, China, of novel coronavirus-infected pneumonia. $\mathrm{N}$ Engl J Med 2020;382:1199-207. doi: 10.1056/NEJMoa2001316.

5. Zhao S, Zhong Z, Xie X, Yu Q, Liu J. Relation between chest CT findings and clinical conditions of coronavirus disease (COVID-19) pneumonia: a multi center study. AJR Am J Roentgenol 2020 ;214:1072-7. doi: 10.2214/ AJR.20.22976.

6. Xu X, Yu C, Qu J, Zhang L, Jiang S, Huang D, et al. Imaging and clinical features of patients with 2019 novel coronavirus SARS-CoV-2. Eur J Nucl Med Mol Imaging 2020;47:1275-80. doi: 10.1007/s00259-020-04735-9.

7. Lai CC, Shih TP, Ko WC, Tang HJ, Hsueh PR. Severe acute respiratory syndrome coronavirus 2 (SARS-CoV-2) and coronavirus disease-2019 (COVID-19): the epidemic and the challenges. Int J Antimicrob Agents 2020 Mar;55(3):105924. doi: 10.1016/j.ijantimicag.2020.105924. 
8. Wong HYF, Lam HYS, Fong AH, Leung ST, Chin TW, Lo CSY, et al. Frequency and distribution of chest radiographic findings in COVID-19 positive patients. Radiology 2020;296(2):E72-8. doi: 10.1148/radiol. 2020201160.

9. Shi H, Han X, Jiang N, Cao Y, Alwalid O, Gu J, et al. Radiological findings from 81 patients with COVID-19 pneumonia in Wuhan, China: a descriptive study. Lancet Infect Dis 2020;20:425-34. doi: 10.1016/S1473-3099(20)300864 .

10. Li Y, Xia L. Coronavirus disease 2019 (COVID-19): role of chest CT in diagnosis and management. AJR Am J Roentgenol 2020;214:1280-6. doi: 10.2214/AJR.20.22954.

11. Zhou S, Wang Y, Zhu T, Xia L. CT features of coronavirus disease 2019 (COVID-19) pneumonia in 62 patients in Wuhan, China. AJR Am J Roentgenol 2020;214:1287-94. doi: 10.2214/AJR.20.22975.

12. Koo HJ, Lim S, Choe J, Choi SH, Sung H, Do KH. Radiographic and CT features of viral pneumonia. Radiographics 2018;38:719-39. doi: 10.1148/ rg.2018170048.

13. Franquet T. Imaging of pulmonary viral pneumonia. Radiology 2011;260: 18-39. doi: 10.1148/radiol.11092149.

14. Samir A, El-Nekiedy AAM, Baess AI, Rizk AM. H1N1 viral pneumonia: spectrum of chest HRCT findings. Egypt J Radiol Nucl Med 2016;47:1293301.

15. Faria IM, Zanetti G, Barreto MM, Rodrigues RS, Araujo-Neto CA, Silva JL, et al. Organizing pneumonia: chest HRCT findings. J Bras Pneumol 2015;41:231-7. 
16. Hosseiny M, Kooraki S, Gholamrezanezhad A, Reddy S, Myers L. Radiology perspective of coronavirus disease 2019 (COVID-19): lessons from severe acute respiratory syndrome and Middle East Respiratory Syndrome. AJR Am J Roentgenol 2020;214:1078-82. doi: 10.2214/AJR.20.22969.

17. Ooi GC, Daqing M. SARS: radiological features. Respirology 2003;8 Suppl (Suppl 1):S15-9. doi: 10.1046/j.1440-1843.2003.00519.x.

18. Das KM, Lee EY, Langer RD, Larsson SG. Middle East respiratory syndrome coronavirus: What does a radiologist need to know? AJR Am J Roentgenol 2016;206:1193-201. doi: 10.2214/AJR.15.15363.

19. Pan F, Ye T, Sun P, Gui S, Liang B, Li L, et al. Time course of lung changes at chest CT during recovery from coronavirus disease 2019 (COVID-19). Radiology 2020;295:715-21. doi: 10.1148/radiol.2020200370.

20. Memish ZA, Perlman S, Van Kerkhove MD, Zumla A. Middle East respiratory syndrome. Lancet 2020;395:1063-77. doi: 10.1016/S01406736(19)33221-0. 